REVISIÓN DE TEMA

Recibido: 23/10/2013

Revisado: 23/11/2013

Aprobado: 23/12/2013

\title{
LA CIUDADANÍA Y LA DEMOCRACIA EN LÍNEA ¿NUEVOS DIÁLOGOS PARA LA SOCIEDAD CIVIL MEXICANA?
}

\author{
JUAN JOSÉ LARA OVANDO \\ Facultad de Ciencias Políticas y Sociales, \\ Universidad Autónoma de Querétaro
}

\begin{abstract}
Resumen
El objetivo de este trabajo es identificar algunos de los mecanismos de participación ciudadana y de la gestión de proyectos sostenibles de gobiernos locales orientados a la innovación social, la ciudadanía 2.0 y el gobierno digital. Se propone que estos proyectos deben gestionarse en el marco de una democracia participativa, una mayor interacción con la comunidad a fin de fortalecer el capital social, la rendición de cuentas y la transparencia a nivel local. Se hace una breve revisión de investigaciones, también de autores sobre la sociedad civil y la acción colectiva, así como del marco jurídico sobre ciudadanía para reflexionar sobre aspectos relevantes para el diálogo entre ciudadanía, las OSC y el Estado; así como, la apropiación de las tecnologías generadas por el gobierno digital y experiencias exitosas de implementación. La orientación espacial es sobre México que es donde se observa la acción municipal y la participación ciudadana.
\end{abstract}

Palabras clave: Ciudadanía, participación, representación, gobernanza, corresponsabilidad, TICs.

\section{ABSTRACT}

The objective of this work is to identify some of the mechanisms of citizen participation and the management of sustainable projects of local governments oriented to social innovation, citizenship 2.0 and digital government. It is proposed that these projects should be managed within the framework of participatory democracy, greater interaction with the community in order to strengthen social capital, accountability and transparency at the local level. A brief review of researches, also of authors on civil society and collective action, as well as the legal framework on citizenship to reflect on aspects relevant to the dialogue between citizens, CSOs and the State; as well as the appropriation of the technologies generated by the digital government and successful implementation experiences. The spatial orientation is about Mexico, where municipal action and citizen participation are observed.

Key words: Citizenship, participation, representation, governance, co-responsibility, ICT. 


\section{Introducción}

El objetivo del artículo es identificar algunos de los mecanismos de participación ciudadana y de la gestión de proyectos sostenibles de gobiernos locales, particularmente aquellos orientados a la innovación social, la ciudadanía 2.0 y el gobierno digital. Este trabajo aun se considera en construcción debido a que todavía falta abundar en la literatura para reflexionar sobre otros aspectos relevantes para el diálogo entre ciudadanía, las OSC y el gobierno municipal, así como la apropiación de las tecnologías generadas por el gobierno digital que no fueron abordados en este primer acercamiento, igualmente para considerar las limitaciones de la participación ciudadana, particularmente la brecha digital para la implementación de proyectos de este tipo.

Se plantea que los espacios públicos, virtuales y físicos, así como la integración de consejos consultivos locales pueden ser un mecanismo que fomente el diálogo para la retroalimentación de los proyectos, así como facilitadores de la rendición de cuentas y la transparencia. Igualmente, se discurre que la participación de la ciudadanía y las OSC en los proyectos municipales no sólo apoyan la colaboración inter-institucional y la innovación social, sino la apropiación de la tecnología y la sostenibilidad de los proyectos en el largo plazo. Finalmente, se añaden observaciones con respecto al uso de las tecnologías.

\section{La democracia y el desarrollo es una tarea de todos}

De acuerdo con datos de Transparencia Internacional (2012), México se encuentra en el lugar 105 del índice de percepción de la corrupción con una calificación de 34. Esta calificación se aproxima a un alto grado de corrup- ción percibida por la ciudadanía, en la que las instituciones requieren ser más transparentes y los servidores públicos más confiables. Los estados-nación que son regidos bajo el modelo político democrático, como México, se sostienen y gestionan gracias a sus instituciones, las cuales son administradas por servidores públicos. Algunos servidores públicos son electos democráticamente y se espera que el resto sea con base a la meritocracia, mas cualquiera tiene la obligación de representar los intereses de la población y estar capacitado y facultado para realizar sus funciones. Sin embargo, prácticas corruptas, mala gestión, falta de transparencia, desigualdad ante la ley por grupos vulnerables, incumplimiento de las promesas de campaña, entre otras razones han mermado el respeto, la legitimidad y la credibilidad tanto de las instituciones como de sus servidores públicos.

La corrupción puede ocurrir en cualquier parte. Cuando los políticos colocan sus propios intereses sobre los públicos. Cuando los oficiales demandan dinero y favores de los ciudadanos por servicios que debieran ser gratuitos. La corrupción no es un sobre con dinero, sino decisiones que afectan nuestras vidas (Transparency International, 2012, 1).

No basta un sentimiento de injusticia para que una sociedad pueda transformarse a sí misma. Son los ciudadanos los que legitiman o deslegitiman las prácticas institucionales; como indica Lawrence Lessig, profesor de leyes en Harvard, los ciudadanos y no sólo los políticos son los corresponsables de la democracia. La práctica democrática no sólo permite elegir a los votantes (democracia representativa), sino que los ciudadanos ejerzan su influencia sobre las decisiones públicas (democracia participativa) y que sus opiniones sean tomadas en cuenta de forma sistemática 
a partir consultas ciudadanas, monitoreo $\mathrm{y}$ otras formas de consenso (democracia deliberativa). Igualmente, que participen en el desarrollo e implementación de programas y medidas ante las problemáticas que los aquejan, sobre todo a nivel local. De acuerdo con Rodríguez (2007), la sociedad civil es corresponsable en el diseño, la elaboración, la promoción y el impulso de los planes e interfaces del desarrollo regional integral por lo que deberá contribuir y colaborar con los proyectos impulsados por el sector público y privado que colabora con fundaciones nacionales e internacionales.

\section{La sociedad civil con y sin fines de lucro}

La noción de sociedad civil es polémica dada la diversidad de perspectivas teóricas y nociones sobre el rol del Estado, gobernanza ${ }^{1}$ y los sistemas políticoeconómicos. Este artículo no intenta recorrer las distintas nociones de Estado, su rol ni los sistemas político-económicos actuales. Sin embargo se comparte que derivado del entendimiento que se tenga del papel y los límites del Estado, así como el marco legislativo se distinguirán los colectivos que componen al tercer sector y la menor o mayor participación de la población (Batta, 2008), así como que se entiende por ciudadanía.

Para fines de la investigación se considera que la sociedad civil es la esfera de relaciones entre individuos, grupos y organizaciones que se desarrollan fuera de las relaciones de poder (Bobbio, 2006) y que integra a individuos y or-

1. Se entiende por Gobernanza, la colección de procesos e instituciones que generan las condiciones para administrar las reglas de ordenamiento y acción colectiva, por ejemplo, los procesos de selección de monitoreo y reemplazo de la administración pública, la capacidad del gobierno para formular e implementar políticas, así como el respeto a los ciudadanos $\mathrm{y}$ a las instituciones que gestionan las interacciones económicas y sociales entre estos (Krishnan y Teo, 2011) ganizaciones con fines de lucro (empresas, cámaras industriales, cooperativas, empresarios, asociaciones) y sin fines de lucro o tercer sector (ciudadanos, agrupaciones formales e informales de individuos). El tercer sector se compone por los agentes privados con fines públicos de distintos sectores y públicos atendidos que construyen relaciones intermedias entre las relaciones de mercado y el Estado. Cada sector de la sociedad civil sin fines de lucro se reconoce por su nivel de especialización, profesionalización, formas organizacionales, fortalecimiento institucional y capital social para incidir y sostenerse en el contexto de un marco regulatorio, los esquemas de competencia por los recursos y las problemáticas atendidas. De esa forma, el tercer sector se reconoce por la búsqueda histórica de soluciones a demandas y necesidades específicas de la comunidad; así como por el trabajo con otros actores de sectores homólogos, gobierno, ciudadanía y empresas.

\section{La ciudadanía}

¿Qué entendemos por ciudadanía? En primera instancia, la ciudadanía se circunscribe al ámbito político de la democracia, a la formación de un Estadonación y a un territorio específico (tiempo, lugar y forma). El Estado-nación refiere a la organización política de la población que comparte una cultura, lengua y un gobierno que sirve a los intereses de la soberanía nacional y se rige bajo un sistema político así como al apego a un marco jurídico, legislativo y administrativo. Por ende, la ciudadanía en la praxis es contingente a una época, problemáticas y cultura de una localidad o región.

En segunda instancia, la ciudadanía es una comunidad, que en caso de nuestro país es, altamente heterogénea dada la gran diversidad de culturas, len- 
guas, etnias y problemáticas regionales y municipales, así como de los grandes contrastes de riqueza-pobreza. Es por ello necesario incorporar a la comunidad y sus vínculos inter ideológicos, inter generacionales, inter disciplinarios, culturales, políticos para solucionar de manera corresponsable y participativa las problemáticas específicas.

En tercer instancia, ciudadanía va más allá del activismo, si bien éste puede fomentar el interés por lo que sucede en otros lugares, no revela su complejidad (Batta, 2008). Numerosas plataformas de redes sociales promueven el activismo virtual en causas específicas independientemente de su locación. Si bien, esto promueve la idea de la aldea global, la ciudadanía política se restringe a su contexto local-regional-nacional. Las plataformas de redes sociales pueden apoyar en la comunicación, así como con los procesos de rendición de cuentas y transparencia, no obstante es sólo una herramienta del rol ciudadano.

En cuarta instancia, en México la ciudadanía es un derecho y una obligación circunscrita en el Capítulo IV de la Constitución Política de los Estados Unidos Mexicanos. En los artículos 35 y 36 constitucionales ${ }^{2}$ se tratan los derechos y obligaciones de los ciudadanos mexicanos que pueden removerse bajo ciertas circunstancias $^{3}$. Este derecho se vincula con la buena gobernanza (rendición de cuentas y transparencia) y la libertad

2. Se mencionan como obligaciones del ciudadano las siguientes: manifestar sus propiedades e información sobre su forma de ganarse la vida al catastro de la municipalidad, inscribirse en el Registro Nacional de Ciudadanos, alistarse a la Guardia Nacional, votar en las elecciones y en las consultas populares, desempeñar los cargos de elección popular de la Federación o de los Estados, y desempeñar los cargos concejiles del municipio en que resida, las funciones electorales y las de jurado.

3. Asimismo, por estar sujeto a un proceso criminal que requiera prisión o en cumplimiento del mismo; por vagancia o ebriedad consuetudinaria, por estar prófugo de la justicia o por sentencia ejecutoria. de expresión y comunicación (Velasco, 2013). El ciudadano podrá expresarse en tanto que existan las condiciones para hacerlo, por ejemplo el libre flujo de información y expresión sin represalias posteriores, así como los medios, canales y espacios para participar de manera libre en la vida política del país.

Finalmente, la ciudadanía considera la gobernanza de un país. Entonces, la acción ciudadana no se restringe al ámbito político, sino que su acción colectiva se entiende en la esfera de lo público y del bien común. El bien común relacionado con temas de salud física y mental, medio ambiente, desarrollo económico y social, calidad de vida, vigilancia a las leyes, seguridad, fomento de las artes y deportes, protección a los derechos de equidad, igualdad de oportunidades, libre expresión y acceso a la información pública.

El ciudadano es capaz de intervenir y comprometerse con el diseño, elaboración, promoción e impulso de planes e interfaces del desarrollo regional integral, a partir de aprovechar las instancias que le permitan contribuir en forma corresponsable, al trabajo que por su parte, ya ha impulsado el sector público y el privado en conjunto con otras fundaciones nacionales e internacionales (Mota, 2007, 17).

\section{Interfaces de interacción entre el gobierno y el tercer sector}

En México, la democracia participativa permite que la sociedad civil participe por medio de herramientas como la consulta popular, las candidaturas independientes y la iniciativa ciudadana ${ }^{4}$, las

4. Las iniciativas ciudadanas son una herramienta innovadora en la política mexicana, porque permiten dar voz a la ciudadanía de manera directa. De acuerdo al artículo 71, fracción IV de la Constitución, se requieren $0.13 \%$ de los ciudadanos inscritos en la lista nominal para presentar una iniciativa ciudadana. 
cuales fueron aprobadas en la Reforma política de la Constitución, en 2012. La Organización para la Cooperación y el Desarrollo Económico (OCDE) plantea que las instituciones gubernamentales y la sociedad civil colaboran e interactúan en tres aspectos cardinales: información, consulta y participación. Conforme la tipología de Isunza y Hevia (2006), las interacciones se plasman en seis interfaces: contribución, transparencia, comunicativa, mandataria, transferencia y cogestiva. A continuación, se describirá cada una de las interfaces mencionadas.

\section{a) Interfaz de contribución}

En esta fase, la sociedad civil informa al Estado -mediante supervisores regionales- de las sugerencias, solicitudes, reconocimientos, inconformidades y comentarios sobre la prestación de los trámites de servicios públicos; así como del comportamiento de los servidores públicos federales en el desempeño de sus funciones (Secretaría de la Función Pública-SFP, 2004).

El ciudadano participa al informar y expresarse de manera libre (Rodríguez, 2007) a través de mecanismos de participación ciudadana como las peticiones ciudadanas, la vinculación con las supervisoras regionales, la atención directa, la gestión ciudadana, la solicitud de acceso a información, el usuario simulado, los grupos de enfoque y los estudios de opinión (SFP-2004).

Estas medidas se relacionan con la transparencia y están restringidas por la capacidad ciudadana para poder efectuarlas; es decir, saber que es posible realizarlo y cómo hacerlo. Las personas saben que pueden tener acceso a la información pero desconocen para qué o cómo la utilizarán. Estos mecanismos deben ir acompañados por facilitadores y no sólo por tecnologías.
No obstante, las Tecnologías de Información y Comunicación (TIC) han permitido gestionar y acelerar estos procesos que deben existir e interesar en la gestión pública. El hecho de colocar un sitio de quejas o consultas sin tomarlas en consideración, dar respuesta o hacerlo de manera extemporánea deslegitima las buenas intenciones y disminuye la participación. Por ende, se requiere de un sistema tecnológico y humano para procesar la información, resolver e interactuar con las Supervisoras Regionales de la SFP, Órganos Internos de Control y el resto de los actores sociales.

A fin de agilizar la participación ciudadana e incentivar su involucramiento en el proceso democrático se pueden diseñar mejores encuestas que sirvan como mecanismo eficiente para la adecuada retroalimentación de la opinión ciudadana. Se propone que el diseño debiera contemplar tres aspectos: 1) no dirigir demasiado la respuesta, 2) restringir las opciones sin dar la oportunidad de que la ciudadanía responda otra cosa y sobre todo, 3) posibilitar la consulta de la información necesaria para la toma de decisiones informada.

\section{b) Interfaz de transparencia}

La interfaz de transparencia da cuenta de la información gubernamental que provee el Estado por medio de páginas de internet gubernamentales, informes periódicos, campañas mediáticas, entre otros (Rodríguez, 2007). En México, la Ley de Transparencia y Acceso a la Información Pública -que considera los tratados internacionales ${ }^{5}$ - demarcó un gran avance para la rendición de cuentas.

5. Tratados internacionales como la Convención Interamericana contra la Corrupción de la Organización de Estados Americanos (OEA), la Convención para Combatir el Cohecho de Servidores públicos Extranjeros en Transacciones Comerciales Internacionales (OCDE) y la Convención de las Naciones Unidas contra la Corrupción. 
Antes de su publicación en el 2003, los portales gubernamentales colocaban a disposición del público internauta la información. Además de la brecha digital y generacional, los portales no contaban con sistemas amigables al usuario para encontrar la información. Casi 10 años han pasado y esta situación ha cambiado de sobre manera y para bien. Casi todas las secretarías han mejorado la interacción con los usuarios en sus páginas web, además tienen páginas en las plataformas de redes sociales más visitadas como Facebook y Twitter. Lo cierto es que la mayoría de las páginas sirven para difundir información más que para interactuar con la ciudadanía, no obstante esto puede considerarse un avance en cuanto que dan a conocer sus servicios.

Como lecciones nos queda saber que no basta la disponibilidad de la información en línea o de archivos centralizados, sin tomar en cuenta su acceso, usabilidad, gratuidad y legibilidad libre de tecnicismos que la hagan comprensible para la ciudadanía. A esta lección se añade la necesidad de que la sociedad civil utilice la información y pueda transformarla en iniciativas concretas. Todd Park ${ }^{6}$ destaca que la información es inútil si no puede aplicarse en beneficio público, por lo que motiva a las iniciativas gubernamentales a enrolar (engage) a los participantes desde el principio, así como facilitar la creación de iniciativas de productos y servicios fundamentadas en la información pública (Park, 2012). Faltaría verificar que quienes tengan acceso a la información sea el público de interés de cada una de las entidades locales y federales.

6. Todd Park es Chief Technology Officer de los Estados Unidos de América desde el 2012, bajo el gobierno de Barack Obama. Dirige el Programa de Iniciativas del Gobierno Abierto.

\section{c) Interfaz comunicativa}

La interfaz comunicativa presenta mecanismos de transparencia de la información pública gubernamental y consejos consultivos para facilitar la mutua transparencia de información entre la sociedad civil y el Estado (Rodríguez, 2007). Cada institución puede solicitar la participación de ciudadanos para consejos consultivos, siempre y cuando cumplan con una serie de requisitos y tengan experiencia o sean reconocidos como expertos en el área. Sin embargo, ¿quién y cómo se eligen los consejos consultivos en la práctica? Y, en qué grado el consejo representa los intereses del resto de la ciudadanía es un tema pendiente.

En México, los consejos consultivos se integran en temáticas específicas y no existe una regulación nacional sobre los consejos locales, por lo que en la práctica su eficacia depende de la buena voluntad de los gobiernos locales, así como de la sólida organización de la ciudadanía (heterogénea y representativa) para involucrarse en el proceso de toma de decisiones (ELLA, 2012).

Entre las buenas prácticas internacionales de comunicación se encuentra la iniciativa de Lenguaje ciudadano. Esta iniciativa de origen sueco ha sido aplicada en otros países como Australia, Reino Unido, Canadá, Estados Unidos, España y en México desde 2004. El Lenguaje ciudadano permite una mejor comprensión de documentos normativos (leyes y reglamentos), administrativos (oficios y notas), comunicados (información oficial) y formatos de trámites y servicios que permita a los ciudadanos entender la información, ejercer sus derechos y cumplir con sus obligaciones sin complicaciones y sin la ayuda de intermediarios. De la misma forma, apoya a que los servidores públicos generen documentos que comuniquen de mane- 
ra clara y directa, así como reduzca los errores y aclaraciones posteriores.

De acuerdo con las experiencias de estos países, el lenguaje claro y directo permite: 1) acercar a los ciudadanos con sus gobernantes, 2) mejorar la confianza del ciudadano con sus instituciones, 3) simplificar y agilizar la operación de las instituciones, 4) reducir la corrupción, y 5) fomentar la transparencia y rendición de cuentas (Valdovinos, et al., 2004).

Otra de las iniciativas de la Función Pública es el Monitoreo ciudadano que fortalece "la transparencia en la gestión pública, la rendición de cuentas y la colaboración entre servidores públicos y ciudadanos para la construcción de un gobierno, atento, responsivo y responsable ante las necesidades y demandas de la sociedad" (León y Girón, 2005). El monitoreo ciudadano utiliza los cuestionarios de satisfacción para adecuar su trabajo a las necesidades de la ciudadanía dentro de los límites legales conducentes. Los cuestionarios se aplican de manera personal, por escrito o a distancia (teléfono e internet) así como a través de talleres o cursos de difusión o resolución de inconformidades (SFP, 2004).

La tendencia por la integración de un Gobierno Digital ha influenciado la forma en que se comunican los distintos actores sociales por medio de las TIC. Bajo la esfera pública en línea, se plantea una vinculación más directa, incluyente, participativa, emocional, informada, veloz, frecuente y personalizada con cada ciudadano, libre de intermediarios y el protagonismo de las jerarquías locales (Wellman, Quan-Hasse, Boase, Chen, Hampton, Díaz y Miyata, 2003). Las plataformas de redes sociales han permitido el acercamiento entre los ciudadanos y los políticos, esto ha servido para fomentar la libre expresión y la rendición de cuentas.
La sociedad comienza a comunicarse con más frecuencia e información en tiempo real. Las tecnologías les permiten incrementar la participación y desarrollar identidades colectivas (Hara y Huang, 2013). No obstante, la interacción directa permanece como la columna vertebral para la conformación de vínculos de confianza, identidad y cohesión social.

El contexto del vecindario cambia de una localidad limitada a lo glocal sustentada en redes de individuos con intereses y problemáticas similares (Calzada, 2012). El apogeo de los medios sociales (social media) manifiesta interconexiones virtuales y la incorporación a plataformas utilizadas por redes sociales (social network). El cambio de msn, hi5, Facebook, Viadeo y google circus varía según el uso y el número de conocidos que interactúen. Un mismo individuo suele pertenecer a varias redes con intereses similares, pero los sujetos en ellas varían por lo que su comunidad es personalizada (Wellman, et al, 2003). La ciudadanía se fragmenta en comunidades especializadas como en Twitter y Tumblr. Finalmente, los medios sociales también sirven para aumentar las relaciones existentes (Robertson y Vatrapu, 2010).

Los ciudadanos pueden vincularse con más personas, más rápido y con más información que antes por lo que una noticia suele difundirse con más velocidad (Wellman, et al, 2003). El incremento de los ciudadanos en los medios y medios sociales va aunado al aumento de la presencia de políticos en línea por medio de páginas sofisticadas, herramientas de comunicación y campañas, que en el caso de México, aun no son reguladas. Los medios sociales han servido para la recaudación de fondos, la diseminación de mensajes, la creación de grupos y la 
movilización entre similares (Robertson y Vatrapu, 2003).

Siguiendo la línea de Habermas y de la psicología política, Mota (2007) enfatiza en la posibilidad del diálogo, del debate y la interlocución como mecanismos de participación social exitosos porque permiten que la comunidad: a) se identifique con un contexto y una problemática compartida; b) transforme su contexto y prácticas en pro del beneficio común; c) genere el cambio institucional de forma participativa, corresponsable y pacífica, y; d) se comprometa y haga corresponsable a quién porta y procura una voz propia.

\section{d) Interfaz mandataria}

La interfaz mandataria refiere a los mecanismos de democracia directa como el referéndum, plebiscito e iniciativas populares, así como la contraloría social (Rodríguez, 2007). Con relación a la democracia directa, la regulación digital permite producir, refinar, deliberar acerca de la legislación y los reglamentos promulgados por las agencias gubernamentales y los órganos reguladores (Robertson y Vatrapu, 2010).

La Dirección Social de Operación Regional y Contraloría Social, de la Secretaria de la Función Pública, describe cuando menos cinco mecanismos de participación social para la contraloría social (SFP, 2004).

En el primer mecanismo se forma universitarios en contraloría social mediante el reclutamiento y capacitación de estudiantes de nivel técnico y superior en actividades de promoción y apoyo a la contraloría social. Como resultados, destaca la diversificación de la participación social y la extensión en la cobertura de las actividades de capacitación, difusión y verificación de obras y acciones. Las tecnologías han permitido facilitar la transparencia de este programa y colocar a disposición de la comunidad un vínculo más directo a través de las plataformas de redes sociales y de una nueva página de internet.

El segundo mecanismo es el programa de escuelas de calidad, en el cual se capacita a servidores públicos, educadores y padres de familia. Se busca incrementar la participación social incluyente, la vigilancia y la evaluación de los recursos económicos asignados en educación. Igualmente, se pretende la corresponsabilidad de los padres de familia en el mejoramiento de la infraestructura física de la escuela y la atención en el desarrollo educativo de los alumnos. Este mecanismo podría emplear e-learning y otros sistemas tecnológicos para mejorar su gestión. Recientemente no se encontraron datos sobre el impacto de este y otros programas.

El tercer mecanismo refiere las campañas de información a la ciudadanía y difusión de la contraloría social. "A los ojos de todos" informa a servidores públicos, población afectada y ciudadanía en general. Las campañas apoyan la transparencia de información (veraz, oportuna y útil) y el uso de recursos públicos, la rendición de cuentas y la disminución de la corrupción. Asimismo, facilitan la participación ciudadana de forma informada, activa y responsable en el control, vigilancia y evaluación de la gestión gubernamental. La difusión se realiza a través de páginas oficiales, blogs, redes sociales y campañas en línea. Actualmente es posible levantar una denuncia en línea, así como dar seguimiento a la misma. En la página de Facebook de transparencia es posible observar las estadísticas que dan cuenta de los resultados de este programa.

El cuarto mecanismo se denomina municipios por la transparencia. En este se imparten talleres de diagnóstico, planeación y profesionalización a servi- 
dores públicos estatales y municipales. El objetivo es integrar una red de capital social de servidores capacitados y líderes para desarrollar estrategias de control preventivo y transparencia basadas en la relación corresponsable gobierno-ciudadanía. Este mecanismo podría mejorarse con el uso de e-learning, comunidades de práctica y el uso de redes de aprendizaje.

Finalmente, la contraloría social en la Estrategia Nacional de Atención a Microregiones realiza reuniones de coordinación con los servidores públicos; constituye y capacita instancias de Contraloría Social en cada Centro Estratégico Comunitario y realiza actividades de verificación de obras, proyectos y acciones. Además, se coordina con otras instituciones y los beneficiarios para agilizar la ejecución, control, vigilancia y evaluación de las obras y acciones en las Microregiones (SFP, 2004). Este mecanismo requiere de gestión institucional tanto como comunicación entre los agentes involucrados. Igualmente, se puede encontrar información en línea, pero es un poco complicado de encontrarla.

e) Interfaz de transferencia

En la interfaz de transferencia es el Estado quién dirige las acciones y proyectos de la sociedad civil por medio de proyectos de desarrollo social, culturales o investigaciones académicas gestionadas por las organizaciones ciudadanas, de instituciones académicas y de contratos específicos.

El marco regulatorio está conformado por la Ley General de Desarrollo Social (LGDS) y la Ley Federal de Fomento a las Actividades Realizadas por Organizaciones de la Sociedad Civil (LFFAROSC). La relación se dirige a las iniciativas de asociaciones, sociedades e instituciones formalmente constituidas, con cierta trayectoria y experiencia en la problemática.
Más recientemente, podemos encontrar algunas iniciativas de desarrollo social destinadas a empoderar a la población al hacerla partícipe de las soluciones. En darles voz, voto y convertirles en agentes de los cambios que ellos mismos requieren. Así como estrategias dirigidas a lograr autosuficiencia, autonomía y pluralidad de manera responsable y propositiva.

Uno de los grandes retos ha sido la gestión de las bases de datos de las organizaciones con relación a su actualización, validación y sistematización de la información más relevante. Los registros de las OSC en Indesol a través de la Clave Única de Inscripción (CLUNI) y en la base de datos del Centro Mexicano para la Filantropía (Cemefi) han servido para identificar la diversidad de sectores, instituciones y objetivos de las OSC. Sin embargo, falta un camino por andar para incrementar la visibilidad del sector, la transparencia y rendición de cuentas, así como la medición del impacto de las mismas. Por lo anterior, proyectos como Civicus y los censos del INEGI permiten incrementar la visibilidad del sector, ya que además de recopilar información la hacen accesible a una audiencia mayor.

\section{f) Interfaz cogestiva}

Esta interfaz mantiene una relación de participación para la dictaminación de proyectos sujetos a recibir recursos públicos y con consejos de carácter vinculatorio como el IFE (Rodríguez, 2007). Las evaluaciones se delimitan al dictamen de los consejos consultivos, no obstante habría que analizar la pertinencia de revisiones por parte de poblaciones más amplias en casos específicos. Por ejemplo, a través de las consultas ciudadanas o las encuestas de satisfacción de la población atendida por la sociedad civil. En este sentido, el contacto con los públicos podría medir el 
impacto social que tienen los proyectos en el corto, mediano y largo plazos. La sistematización de los mismos fortalecería la gestión pública y la rendición de cuentas de las OSC basadas en datos válidos y confiables sobre incidencia, impacto y transparencia.

En suma, es facultad del gobierno: a) facilitar el acceso, la consulta y el libre flujo de información pública sin censura y de manera incluyente, gratuita, legible y accesible; b) gestionar la interlocución con los intereses y las necesidades de la ciudadanía; c) habilitar los espacios públicos, virtuales y físicos, así como los mecanismos que permitan la participación y la deliberación de la ciudadanía, a partir de razones informadas, en las decisiones de interés público. Por ende, el desarrollo tecnológico va de la mano del desarrollo y el fortalecimiento institucional tanto de los municipios como de las OSC.

\section{Comentarios finales}

\section{Los consejos consultivos y los espacios públicos}

Distintos Think Tanks de la región investigan sobre la efectividad de la participación ciudadana, el marco regulatorio y el contexto a fin de recobrar las lecciones aprendidas en la construcción de la democracia y el desarrollo. Algunos de los estudios que parecen más interesantes fueron las propuestas de Fundar y ELLA a través de sus breviarios de política. Estudiar éstas y generar las prácticas relacionadas nos permitiría encontrar el balance y las áreas de oportunidad requeridas para la construcción de políticas y programas a nivel local. En las lecciones aprendidas destaca la necesidad de la buena voluntad política a nivel local para permitir la aplicación efectiva de los mecanismos de participación ciudadana explicados anteriormente. Además, aunque el marco legal del país requiere ciertas modificaciones para facilitar la participación ciudadana, es corresponsabilidad de éste el que se haga escuchar su voz.

Es necesario que los proyectos propuestos por la ciudadanía, las OSC o los consejos consultivos sean adecuados a la localidad a fin de que puedan implementarse de forma sostenible e independiente al cambio de gobierno municipal. Igualmente, que estos programas consideren la capacidad del gobierno local, así como las herramientas tecnológicas disponibles y adecuadas para la región (Kauffman, 2005).

\section{Corresponsabilidad y la formación de una agenda social}

La sociedad -como actor protagónico- se involucra en dar solución a problemáticas cuyos efectos la aquejan, o bien que mejorarán, directa o indirectamente, su calidad de vida y bienestar económico y sociocultural. La participación ciudadana en la democracia pretende contribuir a la toma de decisiones gubernamentales que le afectan, sin necesidad de formar parte de la administración pública o de algún partido.

La corresponsabilidad, como responsabilidad compartida, posibilita la gestión, supervisión y vigilancia a largo plazo de los proyectos en el gobierno local -a través de interfaces- e igualmente aproxima la gobernanza a las necesidades y las problemáticas reales de la población: pues “... sin acción civil e interlocución corresponsable, la gobernabilidad democrática, [la] autonomía y [la] diversificación productiva es insostenible" (Mota, 2007, 15).

No obstante, "se requiere de un tiempo específico y sobre todo, de una propuesta eficaz, viable, deseada y factible colectivamente, para que una comunidad sea capaz de resolver sus problemas en forma compartida" (Mota, 2007, 14). 
Al respecto, Mota, propone la integración de una agenda social que de voz y documente la problemática compartida por los miembros de una comunidad. Que los inmiscuya, motive y empodere para participar en la solución de la problemática identificada. "Documentar nuevos problemas y también formular nuevas propuestas, capaces de articular una nueva agenda que le otorgue validez propia, es el antecedente para incidir participativamente, en el mejoramiento de todos los contextos de la vida diaria" (Mota, 2007, 14-15).

Demasiadas voces y en competencia pueden poner en peligro los derechos y la libertad, así como impedir la gobernabilidad y el pluralismo. De forma paralela, demasiada participación puede reducir la calidad del diálogo, socavar los procesos de gobernanza y retrasar la madurez del gobierno digital; de ahí la necesidad de que las instituciones puedan manejar múltiples voces y propuestas contradictorias a través de los procesos de infraestructura de la información y el desarrollo del gobierno digital (Krishnana y Teo, 2012).

A lo anterior, se añade el reconocer que "la civilidad es portadora de reconocimiento y a la vez identificación del otro, de su alteridad y de su diversidad" (Mota, 2007, 15). Por ende, la independencia de las instituciones y de los órganos de control permite la acción en pro del bien común.

\section{Ciudadanía 2.0}

Como se comentó anteriormente, los espacios públicos, virtuales y físicos, pueden ser un mecanismo que permita la interlocución entre la ciudadanía, las OSC y el gobierno local ya sea a través de los consejos consultivos o de una interacción más directa. Aunque los consejos sólo plantean las políticas y no las implementan, si son entidades de vigilancia que facilitan la retroalimentación de la eficacia de los proyectos, la rendición de cuentas y la transparencia.

Se reitera la posibilidad de la participación ciudadana y las OSC en los proyectos municipales a fin de que los programas puedan implementarse en el menor tiempo posible y sean sostenibles. Si bien el uso intensivo de la tecnología no es un fin en sí mismo, si representa una oportunidad para la sostenibilidad de los proyectos a largo plazo. Sin embargo, destaca la falta de apropiación de la tecnología y la formación de una cultura ciudadana, así como la brecha digital que genera una forma de exclusión social que puede dificultar a los ciudadanos ejercer sus derechos y cumplir sus obligaciones. No todas las regiones del país cuentan con una infraestructura tecnológica, ni con el desarrollo y la madurez institucional necesarias para facilitar la democracia digital.

Además, de acuerdo con Robertson y Vatrapu (2010), el gobierno digital requiere de proveer no sólo los distintos servicios que ya realiza sino de facilitar el acceso tecnológico a los ciudadanos, generar la confianza y seguridad en el uso de sus servicios, así como la facilidad para el uso de sus sistemas en tiempo real y con información actualizada. El gobierno local bien empleado no sólo facilita los procesos de transparencia y democracia digital, sino da el soporte a las comunidades y los vecindarios para auto organizarse en su propio ámbito.

¿Es posible referirse a la ciudadanía 2.0 actualmente en México? Al parecer sí, no sólo debido al uso de la tecnología a nivel municipal, sino por la presencia de ciudadanos que utilizan las herramientas a favor de participar en la vida política del país, de enterarse sobre lo que sucede y actuar en consecuencia. Se infiere que aunque la ciudadanía es precursora de la ciudadanía 2.0, también esta influye sobre la primera. 


\section{BIBLIOGRAFÍA}

Batta Fonseca, V. (2008). Altermundismo: ¿sociedad civil global o nuevo comportamiento antisistémico?, 3 (2): 159-194. En http://www.scielo.org.mx/scielo.php?script=sci_arttex\&pid=S-1870-

Bobbio, Norberto (2006). Estado, gobierno y sociedad. Por una teoría general de la política, FCE, México, 158 pp.

Calzada, I. (2012). “Tecnología, territorio y capital social” en III Congreso Internacional de Tecnología Digital: Aprendiendo a desaprender, Euskadi, 8 y 9 de mayo http://www.congresociudadaniadigital.com

Cemefi e Iniciativa Ciudadana (2011). Una fotografía de la sociedad civil en México. Informe analítico del índice CIVICUS de la sociedad civil, Cemefi e Iniciativa Ciudadana, México, 293 pp.

Comisión Nacional de Derechos Humanos, Derechos humanos en http://www.cndh,org,mx/Cuales_Son_Derechos_Humanos

Diario Oficial de la Federación, Constitución Política de los Estados Unidos Mexicanos, en http://www.diputados. gob.mx/LeyesBiblio/pdf/1.pdf

ELLA (2012). Increasing citizen participation in local governance: Latin American local's citizen councils, policy brief, july 1:6 en http://ella.practiclaction.org/node/1033\#sthash.4Rbvg.KYl.dpuf

Hara, N y Huang B.Y. (2013) "Online social movements", Annual Review of Information of Science and Technology, vol. 45, Chapter 10: 489-522.

Isunza, E y Hevia, F. (2006). Relaciones sociedad civil-Estado en México, Cuadernos para la democratización, CIESAS-UV, Xalapa, $40 \mathrm{pp}$.

Kauffman, E. (2005). E-citizenship, good governance and ICT, Document for the Regional Consultation of the PanAmerican Program, International Development Research Centre, www.idrc.ca/lacro

Krishnan, S. y Teo, T. (2012). "Moderating effects of governance on information infrastructure and e-government development" en Journal of American Society for Information Science and Technology, 63(10): 1929-1946 en http://onlinelibrary.wiley.com/doi/10.1002/asi.22660/full

León M., A.M. y Girón B., S. (2005). Guía para el monitoreo ciudadano, Unidad de Vinculación para la Transparencia, SFP y Consejo Ciudadano para la Transparencia, México en http://portal.funcionpublica.gob.mx:8080/ wb3/work/sites/SFP/resourses/LocalContent/144

Mota B., G.A. (2007).“Educación ciudadana y construcción participativa de la autosuficiencia para el desarrollo integral a través de la gestión y el diseño de políticas públicas" en VII Seminario Anual de Investigación sobre el Tercer Sector en México: Hacia una cultura de ciudadanía y rendición de cuentas, UAM, México.

Park, T., (2012). "Unleashing the power of innovation in government" en Social Good Summit, ONU, New York en http://socialgoodsummit2012,eventbrite.com/

Robertson, S.P. y Vatrapu, R.K. (2010). “Digital goverment” en Annual Review of Informatio Sciencie and Technology, Cronin, vol. 44, chapter 8: 317-364.

Rodríguez C., M.A. (2007). "Percepciones sobre el monitoreo ciudadano como herramienta para la rendición de cuentas del gobierno federal" en VII Seminario Anual de Investigación sobre el tercer Sector en México: hacia una ciudadanía y rendición de cuentas, UAM, México.

Secretaría de la Función Pública (2004). Mecanismo de participación ciudadana. Información histórica de las obligaciones de transparencia de la SFP, SFP, México.

Transparency Internacional (2012). Corruption perceptions. Index 2012. En www.transparency.org

Valdovinos C, C.M., Quintana M., R.J., Mares C., A.A., Maldonado V., F., Maldonado R., O., Pontigo S., F.J., Sierra F., S., Ramírez V., D., Iglesias C., C., Pérez M., A. y Block, S. (2004). Lenguaje ciudadano: Un manual para quién escribe en la Administración Pública Federal, Dirección General de Simplificación Regulatoria, SFP, México.

Velasco, E., M.N. (2013). "Mecanismos corresponsables de colaboración ciudadana en la e-democracia” en Valentino Morales L. y José Eduardo Moreno F., Estrategias y metodología para el gobierno digital en municipios mexicanos, Infotec, México, pp. 53-55.

Wellman, B., Quang-Hasse, A., Boase, J., Chen, W., Hampton, K., Díaz, I. y Miyata, K. (2003). “The social affordances of the internet for networked individualism" en Journal on computer-mediated communication, $8(0)$. 